\title{
Temporal organization of ongoing brain activity
}

\section{Journal Article}

\section{Author(s):}

Lombardi, Fabrizio; De Arcangelis, Lucilla

Publication date:

2014-10

Permanent link:

https://doi.org/10.3929/ethz-b-000383756

\section{Rights / license:}

In Copyright - Non-Commercial Use Permitted

\section{Originally published in:}

The European Physical Journal Special Topics 223(11), https://doi.org/10.1140/epjst/e2014-02253-4 


\title{
Temporal organization of ongoing brain activity
}

\author{
F. Lombardi ${ }^{1, a}$ and L. de Arcangelis ${ }^{2}$ \\ 1 Institute of Computational Physics for Engineering Materials, ETH, Zurich, Switzerland \\ 2 Department of Industrial and Information Engineering, Second University of Naples, \\ INFN Gr. Coll. Salerno, Aversa (CE), Italy
}

Received 20 May 2014 / Received in final form 18 August 2014

Published online 24 October 2014

\begin{abstract}
Ongoing brain activity results from the mutual interaction of hundred billions non-linear units and represents a significant part of the overall brain activity. Although its complex dynamics has been widely investigated, a large number of fundamental questions are still open, many of them concerning its temporal structure. Why does a certain population of neurons fires synchronously? Are these synchronized bursts following each other randomly or are they correlated according to some organizing principle? Far from addressing the fundamental problem of its functions, in the present article we focus on the problem of temporal correlations of ongoing cortical activity. We first overview the major features of its temporal structure and review recent experimental results, with particular emphasis on alternative approaches inspired in the theory of stochastic processes; then we introduce a neuronal network model inspired in self organized criticality and compare numerical results with experimental findings.
\end{abstract}

\section{Introduction}

Since the early days of electroencephalography (EEG) the ongoing brain activity has attracted a considerable interest. The observation of alpha waves, an 8 to $12 \mathrm{~Hz}$ rhythm reported by Berger in the 1929 [1], was followed by intensive research, which led to the identification of many others oscillatory patterns during rest, sleep and successively under anesthesia [2]. Oscillations are basically driven by the synchronous firing of a large population of neurons and represent only one, even though the most surprising probably, of the spatio-temporal organization forms of spontaneous activity. More recently, ongoing cortical activity has been intensively investigated using techniques as different as magnetoencephalography (MEG), functional magnetic resonance imaging (fMRI), local field potential (LFP) and single units recordings. Beside oscillatory patterns, new forms of spatio-temporal organization have been identified, opening new perspectives towards the understanding of its functions. In particular, discovery of neuronal avalanches and observation of universal scaling features have

${ }^{a}$ e-mail: fabrizio.lombardi@ifb.baug.ethz.ch 
provided the first experimental evidence for a picture of the brain as a system acting in a critical state [3-5], thus suggesting a new approach based on the theory of critical phenomena [6-9]. Firstly identified in the LFPs of small cortical cultures [3], avalanches have been recently found in the resting MEG of human brain and in the fMRI blood oxygenated dependent (BOLD) signal [7]. In this case a novel approach to the analysis of fMRI data, which consists in reducing BOLD signal to a point process, has also allowed to define an order parameter and show that the resting brain is mainly acting around an order-disorder phase transition.

In the following we will review recent experimental findings on ongoing cortical activity, focusing on its temporal structure. The analysis of power spectra has had a predominant role in the investigation of temporal features, especially periodic oscillations and long range correlations [10-15]. However, in recent years, alternative approaches based on the theory of stochastic processes have been proposed, attempting to address more fundamental questions about the temporal organization of spontaneous brain activity. For instance quantities as the waiting times, largely used in the investigation of stochastic natural phenomena $[16,17]$, have been introduced in the analysis of synchronized bursting events [18] and neuronal avalanches $[19,20]$ in order to characterize their temporal organization. At the same time the discovery of neuronal avalanches with their scale free statistics has led to the formulation of a novel numerical model inspired in self organized criticality (SOC) [19,21,22]. We will review results from this model and compare them with experimental findings.

\section{Ongoing activity: Temporal structure}

Ongoing activity can be viewed as a sequence of bursts or up-states, periods of sustained spiking activity in a large population of neurons. Up-states usually last from a few to several hundreds of milliseconds and are separated by periods of substantial inactivity, called quiescent periods or down-states, and lasting up to several seconds [23-25]. In the most general case this alternation of high activity and quiescence does not show a clear periodic character and some authors refer to it as arrhythmic brain activity [26]. However, during sleep or under anesthesia, as well as in vitro, it clearly appears as slow oscillations with frequencies ranging in the interval $0.1-1 \mathrm{~Hz}$.

Taken as a whole, a sequence of up-states can be viewed as a stochastic process and studied accordingly. A first characterization in this sense is due to Segev et al. [18]. Considering in vitro neuronal networks of different sizes and recording their spiking activity with a microelectrode array (MEA), they have studied the distribution of jumps in the sequence of waiting times, namely the quantity $\Delta(i)=\Delta t(i+1)-\Delta t(i)$, where $\Delta t$ is defined as the time interval between two successive bursts. They have found that this distribution can be approximated by a symmetric Levy distribution. Moreover the power spectral density (PSD) exhibits a power law decay for low frequencies, regardless of the network size, indicating long range temporal correlations in bursting activity.

A quantity widely used in the investigation of stochastic natural phenomena and able to discriminate between a simple Poisson and a correlated process is the distribution of waiting times. In the first case this distribution is indeed an exponential, whereas it exhibits a more complex behavior with a power law regime if correlations are present. The distribution of waiting times has been recently employed to characterized the temporal organization of neuronal avalanches in cultures of rat cortex $[19,20]$. Avalanches are cascades of activity characterized by a scale free distribution of size and duration [3-5,27-29], typical features of systems in a critical state. In [19], neuronal avalanches are identified in the local field potentials (LFP) of spontaneous cortical activity. Significant negative LFP deflections (nLFPs) are detected 
by a threshold crossing criterion. The threshold is set at three times the standard deviations (SD) of the LFP fluctuations calculated for each electrode. Then, neuronal avalanches are defined as spatio-temporal clusters of nLFPs on the microelectrodes array (MEA) [3,19]. For each avalanche $j$ its starting and ending times, $t_{j}^{i}$ and $t_{j}^{f}$, are identified and the distribution $P(\Delta t)$ of waiting times $\Delta t_{j}=t_{j+1}^{i}-t_{j}^{f}$ between successive avalanches is analyzed. A power law behavior at short time scales, namely from few milliseconds to $200-300 \mathrm{~ms}$, is found for all the experimental samples, indicating that avalanches are temporally correlated if sufficiently close in time, which requires a sustained synaptic and firing activity in the network, namely an up-state. Therefore up-states can be seen as clusters of avalanches. For longer waiting times the distribution is generally characterized by a local maximum which in many cases leads to a peculiar non-monotonic behavior. Longer waiting times correspond to quiescent periods and are simply waiting times between successive up-states. Accordingly, in [20] an up-state (down-state) is defined as a consecutive series of avalanches separated by $\Delta t$ shorter (longer) than the longest $\Delta t$ falling within the power law regime of the distribution $P(\Delta t)$ and waiting times between successive up-states are found to be distributed around a certain value $1 s<T<2 s$, the tail of the distribution being well fitted by an exponential [30]. This behavior indicates that the recurrence of up states show a more or less pronounced characteristic time, reminiscent of slow oscillations. We will discuss this point in more details in the section dedicated to numerical results.

More generally, the observation of characteristic times is related to oscillations which are embedded in the sequence of up-states [26,31]. Nested frequencies have been recently analyzed in the spontaneous EEG signal, revealing a rich temporal organization with the phase of lower frequencies modulating the amplitude of the higher ones [26]. In rat cortex in vivo and in vitro, the spatio-temporal structure of nested ocillations has been found to be equivalent to neuronal avalanches [29]. Indeed the analysis of spontaneous neuronal activity in the rat cortex layer $2 / 3$, indicates that during the second week postnatal bursts develop a temporal organization of higher frequency oscillations, $\beta-(15-30 \mathrm{~Hz})$ and $\gamma-(30-100 \mathrm{~Hz})$, nested into lower frequencies $\theta-(4-15 \mathrm{~Hz})$ oscillations, while the spatio-temporal organization of LFPs is characterized by the scaling behavior of neuronal avalanches [29]. The intimate relationship between neuronal avalanches and oscillations has been also enlighten in [20], by studying the distributions $P\left(\Delta t ; s_{c}\right)$ of waiting times between consecutive avalanches of size larger than a threshold $s_{c}$. In this way smaller events are removed from the avalanche time series and the distribution $P(\Delta t)$ changes as a function of $s_{c}$, showing peaks that were not present in the original $P(\Delta t)$. These appear for values of $s_{c}$ larger than $40 \mu \mathrm{V}$, on time scales where the power law regime is found in the original distribution. The first peak is at $\Delta t \simeq 40-60 \mathrm{~ms}$ and has to be related to the period of $\beta$ oscillations. The second one arises at $\Delta t \in[80 \mathrm{~ms}, 250 \mathrm{~ms}]$ and corresponds to the period of $\theta$ oscillations [20]. This transformation, which consists of removing events from a time series according to their size, is reminiscent of renormalization group in real space [32] and has been used to investigate universal features in earthquake occurrence. In that case the waiting time distributions obtained by setting different magnitude threshold values collapse onto the same universal curve after rescaling $\Delta t$ by the average rate in the time series [16]. This property reveals that the fundamental features of earthquake occurrence do not change under the removal procedure described above. Conversely, in [20] it has been shown that the distributions $P\left(\Delta t ; s_{c}\right)$ do not collapse onto a single curve after rescaling waiting times by the average occurrence rate. However, the waiting time distributions for up and down-states do exhibit universal scaling features: The distributions $P\left(\Delta t ; s_{c}\right)$ for the up-state and for the down-state collapse onto two distinct universal curves when rescaled by $r_{u p}=\langle\Delta t\rangle_{u p}$ and $r_{d w}=\langle\Delta t\rangle_{d w}$ 
respectively. This indicates that ongoing activity is governed by different dynamic mechanisms at short and large time scales [20].

This point will be carefully discussed in the section dedicated to numerical results. For the sake of completeness, we observe that the same analysis has been carried out for spike avalanches in freely behaving (FB) and anesthetized rats (AR) in [33], where the distributions of waiting times have been found to be monotonic. Universal scaling features have been observed for freely behaving rats when waiting times were rescaled by the average occurrence rate, but not for anesthetized rats.

\section{Neuronal network model for ongoing brain activity}

In this section we briefly review a model which includes the fundamental physiological features of neuronal behavior: Firing at threshold, refractory period and longterm Hebbian-like plasticity $[19,21,22]$. We consider $N$ neurons at random positions, characterized by their potential $v_{i}$. Neurons are connected by a scale-free network. More precisely, to each neuron $i$ we assign an out-going connectivity degree, $k_{\text {out }_{i}}$, in agreement with the experimentally measured properties of the functional connectivity network [34]. Choosing different networks does not affect the scaling behavior of avalanche distributions $[21,22,35]$. Once the network of output connections is established, we identify the resulting degree of in-connections, $k_{i n_{j}}$, for each neuron $j$. To each synaptic connection we assign an initial random strength $g_{i j}$, where $g_{i j} \neq g_{j i}$, and to each neuron an excitatory or inhibitory character, with a fraction $p_{i n}$ of inhibitory synapses. Activity is initiated increasing by a small amount the potential of a random neuron. Whenever at time $t$ the value of the potential at a site $i$ is above a certain threshold, $v_{i} \geqslant v_{\max }$, the neuron sends action potentials which arrive to each of the $k_{\text {out }}$ pre-synaptic buttons and lead to a change in the potentials of the post-synaptic neurons proportional to the strength of the synapsis $g_{i j}$,

$$
v_{j}(t+1)=v_{j}(t) \pm \frac{v_{i} \cdot k_{\text {out }}}{k_{i n_{j}}} \frac{g_{i j}}{\sum_{k} g_{i k}},
$$

where the plus or minus sign is for excitatory or inhibitory synapses, respectively, and the sum over $k$ is on all neurons connected to $i$. After firing, a neuron is set to a zero resting potential and in a refractory state lasting $t_{r e f}=1$ time step, during which it is unable to receive or transmit any charge. Activity propagates through connected neurons until no potential is above threshold. The number of firing neurons then represents a neuronal avalanche. At the end of an avalanche, we implement an Hebbian-like plasticity rule: The strength $g_{i j}$ of the used connections is increased proportionally to the membrane potential variation $\delta v_{j}$ of the postsynaptic neuron $j$ induced by the presynaptic neuron $i$ during the avalanche,

$$
g_{i j}=g_{i j}+\left|\delta v_{j}\right| / v_{\max }
$$

whereas the strength of all inactive synapses is reduced by the average strength increase per bond

$$
\Delta g=\sum_{i j} \delta g_{i j} / N_{B}
$$

where $N_{B}$ is the number of bonds. An external stimulus then triggers further activity in the system. Since this plasticity acts on time scales longer than avalanche duration, we apply the plasticity protocol for a given number of stimulations, in order to simulate both long term potentiation (LTP) and long term depression (LTD), and then study avalanche activity without changing synaptic strengths. 


\section{Up-down state dynamics}

We study the transition between up and down states by means of a mechanism relying on the idea that an up state terminates when the system is not able to sustain activity any longer, namely when either the exhaustion of available synaptic vesicles [36] or the increase of factors inhibiting the release [37] cause a sharp transition towards a down state. For simplicity, in simulations we assume that the transition occurs after a sufficiently large discharge event, which causes a sufficiently strong network inhibition. Accordingly, at the end of each avalanche we measure its size in terms of the sum of positive $\delta v_{i}$ of all neurons, $s_{\Delta V}=\sum_{i} \delta v_{i}$. When the last avalanche is larger than a threshold $s_{\Delta V}^{\min }, s_{\Delta V}>s_{\Delta V}^{\min }$, the system transitions into a down state and neurons become hyperpolarized proportionally to their previous activity; namely, we reset

$$
v_{i}=v_{i}-h \cdot \delta v_{i}
$$

This rule models the local inhibition experienced by a neuron, due to spike adaptation [38], adenosine accumulation [37], synaptic vesicles depletion [36] or blockade of receptor channels by the presence of external magnesium [39]. Conversely, if the avalanche just ended has a size $s_{\Delta V} \leqslant s_{\Delta V}^{m i n}$, the system either will remain in or will transition into an up state, which typically happens at the end of a down state. After each avalanche in the up state, all neurons firing are set to the depolarized value

$$
v_{i}=v_{\max }\left(1-s_{\Delta V} / s_{\Delta V}^{\min }\right) \text {. }
$$

This equation states that the neuron potential depends on the response of the whole network via $s_{\Delta V}$, in agreement with measurements of the neuronal membrane potential, which remains close to the firing threshold in the up state [40]. The threshold $s_{\Delta V}^{\min }$ controls the extension of the up state and, therefore, the level of excitability of the system.

The high activity in the up state must be sustained by collective effects in the network otherwise the depolarized potentials would soon decay to zero. Therefore, the stimulation in the up state has a random value in the interval $d_{u}=\left[0, s_{\Delta V}^{\min } / s_{\Delta V}\right)$, whose amplitude depends on past activity through the size of the previous avalanche $s_{\Delta V}$. Conversely, during the down state the stimulation of the system has a small constant amplitude applied at a random site. This drive reproduces the effect of the small depolarizations due to miniature potentials (minis) from spontaneous synaptic release observed in the down state [41]. The drive slowly brings the system back in an up state not correlated to past activity.

Equations (4) and (5) each depend on a single parameter, $h$ and $s_{\Delta V}^{\min }$, which introduce a memory effect at the level of single neuron activity and the entire system, respectively.

\subsection{Temporal organization of avalanches}

In Sect. 2 we have seen that the distributions of waiting times between successive avalanches in cortex slice cultures exhibit a complex non-monotonic behavior with an initial power law regime and a minimum followed by a local maximum. Depending on the experimental sample, this power law regime extends over two or three decades and exhibits an exponent $2.0 \leqslant \mu \leqslant 2.5$, indicating that avalanches are temporally correlated if they are sufficiently close in time, which requires a sustained synaptic and firing activity in the network [19].

In order to investigate the origin of the experimental non-monotonic behavior, we simulate avalanche activity and generate a sequence of up and down states, each upstate being composed of several neuronal avalanches (Fig. 1). As shown in Fig. 2, we 


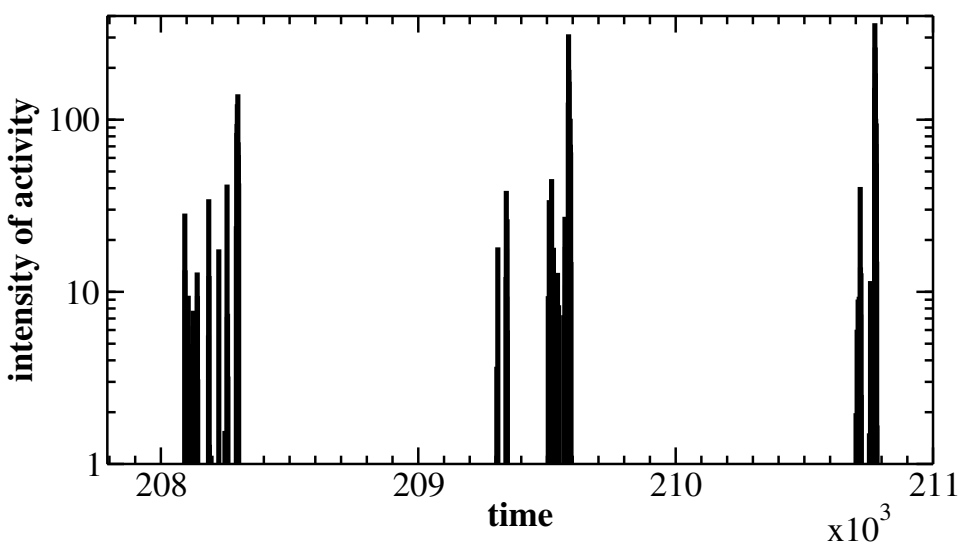

Fig. 1. A short sequence of avalanche activity. Each bar represents an avalanche, whose size is measured in terms of $s_{\Delta V}$. Avalanches are clearly clustered. Each cluster is an up-state, whereas down-states are the time intervals without activity separating successive up-states.

obtain numerical distributions $P(\Delta t)$ of waiting times which exhibit a non-monotonic behavior and a power law regime with the same exponent $\mu \sim 2$ measured experimentally [19]. Insets of Fig. 2 show the contribution to the overall waiting time distribution coming from each of the two states. In the up-states waiting times clearly follow a power law distribution and the good agreement between numerical and experimental curves indeed confirms that it originates from close in time correlated avalanches (Fig. 2, lower insets). Conversely, down-states produce long waiting times mostly contributing to the tail of the $P(\Delta t)$, exhibiting a characteristic value $\tau_{d}$. (Fig. 2, upper insets). This distribution basically coincides with the distribution of waiting times between successive up-states, shown in Fig. 3. We notice here that on average, both numerically and experimentally, waiting times between successive up-states are distributed around $\Delta t \simeq 2000 \mathrm{~ms}$ and the tail of the distribution is well fitted by an exponential [30]. This behavior implies that the recurrence of up states has a more or less pronounced characteristic time.

However this characteristic time is sample dependent. In order to reproduce different observed behaviors, numerically we tune $h$ and $s_{\Delta V}^{\min }$ separately, finding that the ratio $R=h / s_{\Delta V}^{\min }$ provides the best fit between experimental and numerical data if $R \simeq 10^{-4}$ [19]. For instance, increasing the threshold value $s_{\Delta V}^{\min }$ while keeping $h$ fixed, clearly produced a major shift in the data. However a good agreement with the experimental curves can be recovered by increasing $h$ in such a way $R \simeq 10^{-4}[19]$. Therefore $h$ and $s_{\Delta V}^{m i n}$ drive two competing factors, whose balance results in the waiting time distributions measured experimentally. The threshold $s_{\Delta V}^{\min }$ basically controls the amount of excitatory inputs neurons receive between successive avalanches in the up-state. Indeed the stimulation has a random value in an interval whose size depends on $s_{\Delta V}^{\min }$ in such a way the larger is $s_{\Delta V}^{\min }$ the larger that interval and the stronger the stimulation (see Sect. 3). By means of $s_{\Delta V}^{\min }$, we then control the excitation in the system. On the other hand, the parameter $h$, controlling the hyperpolarization locally experienced by neurons after up states, represents a sort of dynamical inhibition. Therefore $R$ has to be interpreted as the ratio between dynamical excitation and inhibition in the network [19]. Indeed altering $R$, not only leads to a major shift of the numerical waiting time distributions from the experimental ones, but also alters the scaling behavior of the avalanche size distributions, which could be related to pathological experimental conditions [3,20,42]. Tuning either of the parameters leads to the optimal value of $R$, namely to an optimal balance between excitation and inhibition. 


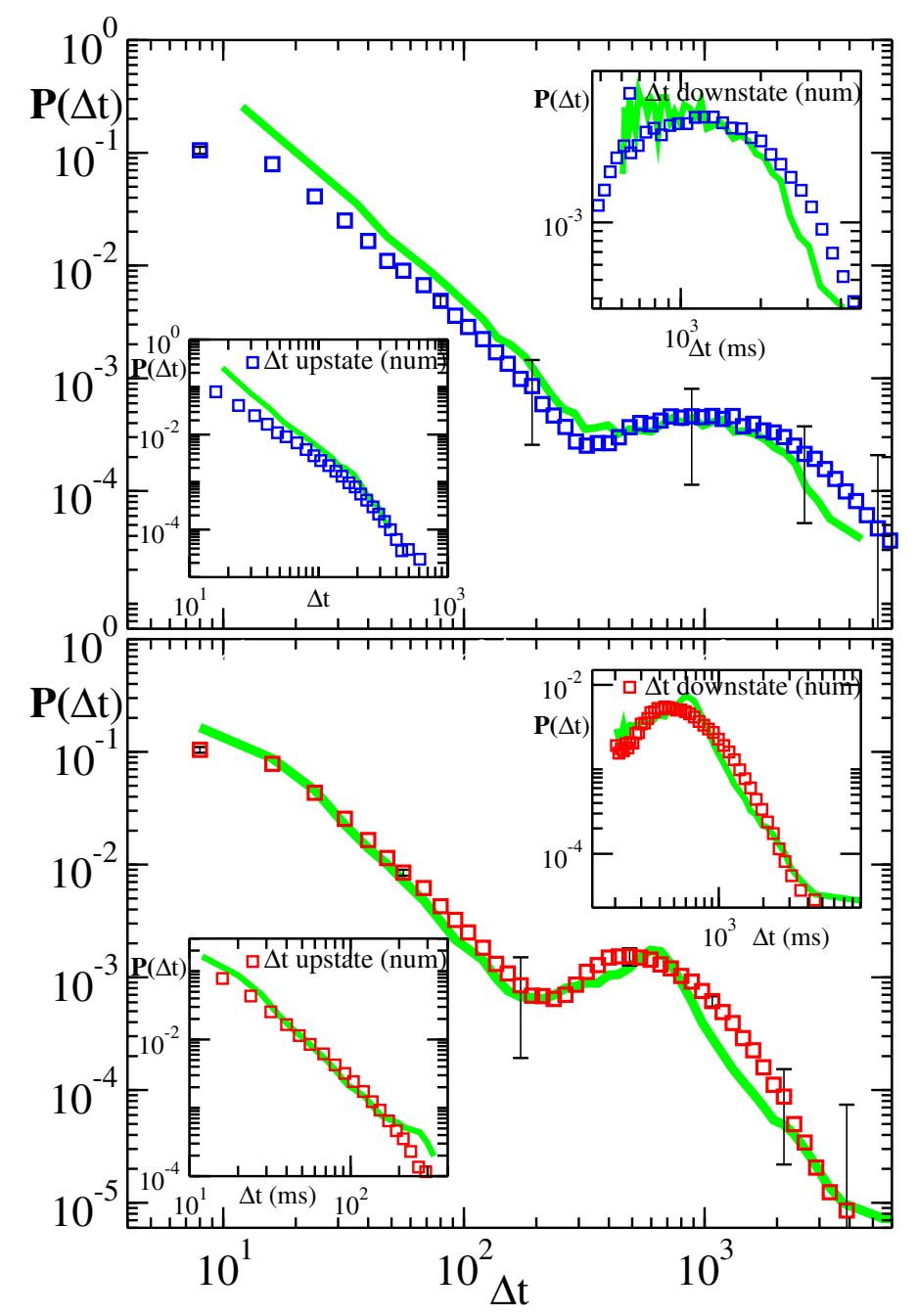

Fig. 2. Waiting time distributions measured in two different experimental samples are compared with the average numerical distributions for 100 networks with $\mathrm{N}=64000$ neurons. In the insets: the waiting time distribution evaluated separately in the up (lower insets) and down state (upper insets) for the numerical (symbols) and the experimental data (green curves). For the numerical curves, statistical error bars are comparable with the symbol size.

\subsection{Universal features of avalanche temporal occurrence}

In [20] it has been pointed out that, for avalanche activity in vitro, the distribution $P(\Delta t)$ is not solely controlled by the average occurrence rate $r=1 /\langle\Delta t\rangle$. The different dynamics governing up and down-states implies that the distributions $P\left(\Delta t ; s_{c}\right)$ of waiting times between consecutive avalanches of size larger than $s_{c}$ do not collapse into a single curve when $\Delta t$ is rescaled by $r$. As previously shown, the waiting time distribution exhibits a very different behavior at short and long time scales, which results from the slow alternation between up and down-states [19,20]. When avalanches are removed from the time series, the power law regime, which reflects clustering in the up-state, and the tail of the distribution, which results from the 


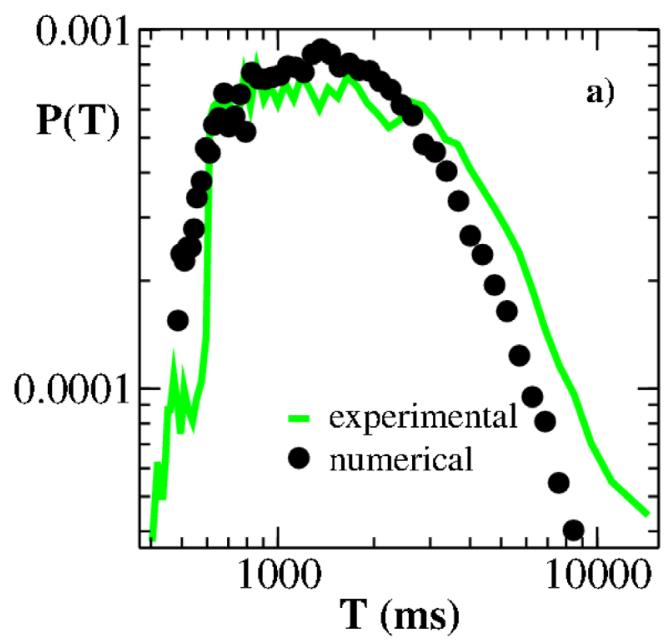

Fig. 3. Distribution of waiting times between successive up-states averaged over 100 configurations of a network of $\mathrm{N}=64000$ neurons with $p_{i n}=0.1$ (black symbols). Green thick curve: experimental data. Figure from [20].

down-state dynamics, change in a very different way [20]. It has been shown that the former is controlled by the average occurrence rate in the up-state, $r=1 /\langle\Delta t\rangle_{u p}$, while the latter is controlled by $r=1 /\langle\Delta t\rangle_{d w}$, the average occurrence rate in the down-state. Therefore the waiting time distributions for this two network states do exhibit universal scaling features. Indeed the distributions $P\left(\Delta t ; s_{c}\right)$ in the up-state and that ones in the down-states collapse onto two distinct universal curves: A power law for the up-state (Figs. 4a, c) and a distribution peaked around a characteristic value for the down-state (Figs. 4b, d) [20].

We have applied the removal procedure described in [20] to the numerical distributions in Fig. 2. We have rescaled the distribution of waiting times in the up state (upper insets in Fig. 2) by $r_{u p}$ and the distribution of waiting times in the down-state (lower insets in Fig. 2) by $r_{d w}$. As shown in Fig. 4, our numerical results are in good agreement with experimental findings.

\section{Discussion}

We have seen that, in a coarse grained description, ongoing activity consists of a sequence of synchronized bursts or up-states, which in many cases emerge as slow oscillations. Bursts are characterized by an intense spiking activity in large cortical areas and alternate with quiescent periods, characterized by a low average spiking rate [23-25,43]. The analysis of their fine temporal structure has unveiled the presence of nested oscillations, showing that the phase of the slower oscillations modulate the amplitude of the faster ones $[26,31]$. On the other hand, it has been shown that bursts are organized in neuronal avalanches and an intimate relationship between their spatio-temporal structure and nested oscillations has been found [20,29]. The scale free statistics of neuronal avalanches has suggested that the brain operates in a critical state, thus arousing a considerable interest among physicists. From the theory of critical phenomena we know that a system acting at the critical point is characterized 

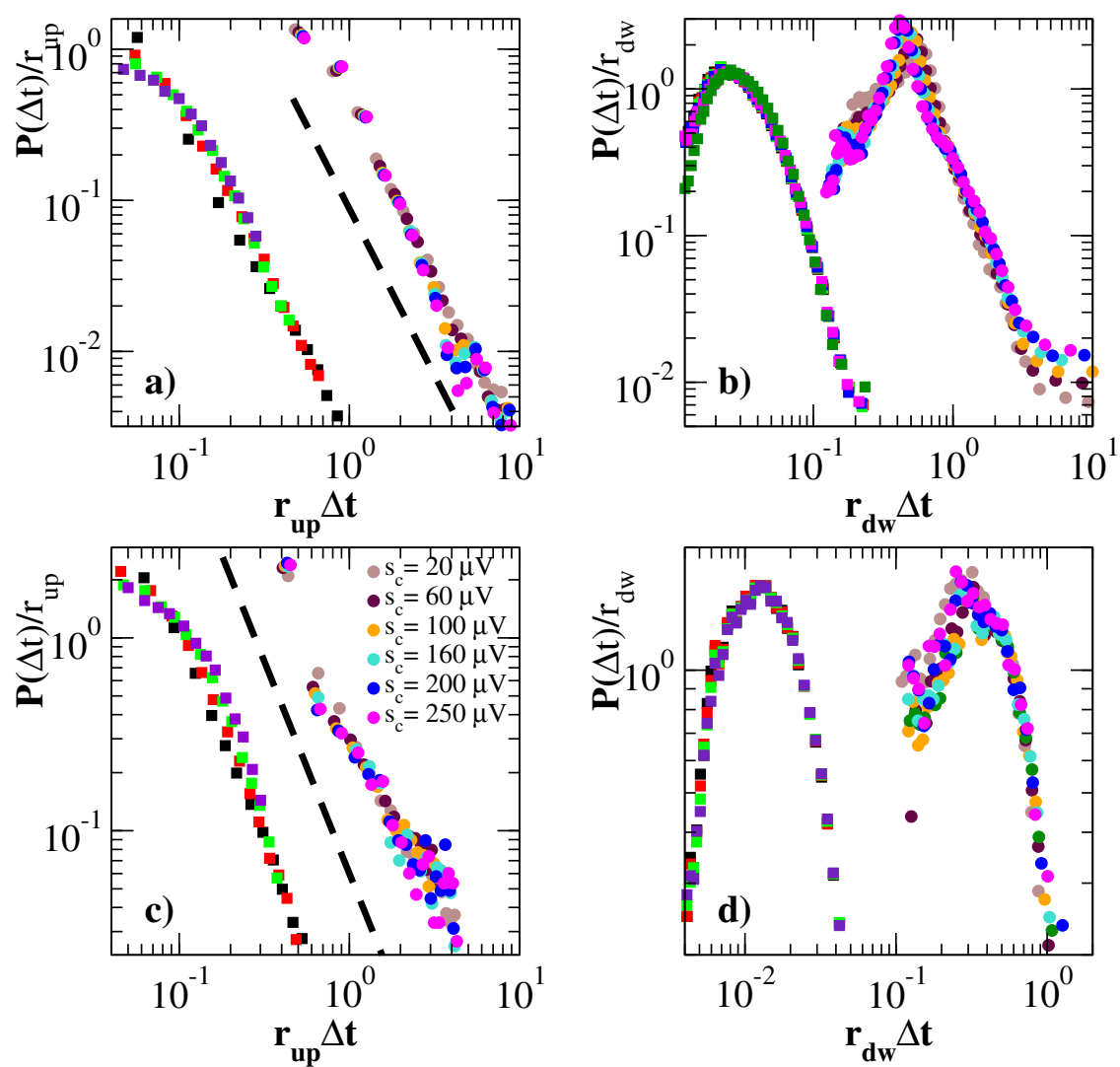

Fig. 4. Distributions of waiting times in the up (a,c) and down-state (b,d) for numerical and experimental data in Fig. 2 and different thresholds $s_{c}$ on avalanche size. (a) Distributions after rescaling by the mean rate in the up-state (Fig. 2, lower panel). Numerical data are shifted to the left. (b) Distributions after rescaling by the mean rate in the down-state (Fig. 2, lower panel). Numerical data are shifted to the left. (c) Distributions after rescaling by the mean rate in the up-state (Fig. 2, upper panel). Numerical data are shifted to the left. (d) Distributions after rescaling by the mean rate in the down-state (Fig. 2, upper panel). Numerical data are shifted to the left. The dashed line represents a power law with exponent -2.2 . Figure adapted from [20].

by long-range spatial and temporal correlations. The power law characterizing the distributions of avalanche size, as well as a correlation length increasing with the cluster size in the fMRI [8], constitutes an indication of the former, whereas a nontrivial power law decay in the frequency spectrum, i.e. $1 / f$, is the fingerprint of the latter. The so called $1 / f$ noise is ubiquitous in nature, from the light of quasars [44] to music [45], and has been observed in the PSD of electrophysiological signals as different as EEG, MEG and LFP $[12,14,15,46]$.

In 1987, Bak, Tang and Wiesenfeld [47] proposed the concept of self organized criticality(SOC) to explain how $1 / f$ noise and long range correlations can spontaneously emerge in processes far from equilibrium. They argued that the dynamics which produces the power laws observed in the steady states of many natural phenomena does not involve any fine tuning of parameters, namely systems self evolve to a state that is at the edge of stability. Such a state then shows long range spatio-temporal correlations similar to those observed in equilibrium critical 
phenomena. The dynamic mechanism of SOC consists in a slow accumulation and a fast redistribution of energy in the system. To exemplify this idea, they introduced the "sandpile" model, alias BTW model. This model exhibits scale free distributions for avalanche size and duration [47] and a power spectrum of the avalanche signal of the form $1 / f^{\beta}$ with $\beta=1.6[48]$.

The observation of neuronal avalanches in 2003 has indeed suggested an interpretation of ongoing cortical activity in term of SOC and, at the same time, has unveiled similarities with other natural phenomena exhibiting scale free behavior, as earthquakes or solar flares. However, while the waiting time distribution in the original BTW model is a simple exponential, for many of these phenomena is not $[16,49,50]$. In particular, for neuronal avalanches this distribution exhibits a peculiar non-monotonic functional behavior: A power law decay at short time scales followed by a local minimum and a peak localized at larger time scales, which is generally followed by an exponential decay $[19,20]$. The power law regime indicates that avalanches close in time are temporally correlated [51], which requires a sustained synaptic and firing activity in the network. Therefore avalanches are significantly correlated during up-states and not on longer time scales, because of the sparse synaptic activity in down-states [23-25]. Several models have been proposed in order to show that SOC-like dynamics can provide temporal correlations among discharge events and a non-exponential distribution of waiting times [52,53]. Here we have shown that our model, inspired in SOC, is able to capture the peculiar, non-exponential and nonmonotonic behavior of the waiting time distribution for neuronal avalanches recorded in cortex slice cultures [19]. Moreover, numerically generated up and down-states, exhibit the same universal features found experimentally [20]. The model suggests that correlations in the up-state originate from network mechanisms, which are crucial to sustain the high activity in a large population of neurons. They act as a form of short-term memory, which gives rise to the initial power law regime in the waiting time distribution. On the other hand, it shows that the synaptic activity during the down-state can be modeled as a random process that slowly brings the system back to the up-state, with no memory of past activity. Indeed, as observed experimentally, the waiting distributions associated to down-states exhibit an exponential tail [20]. This implies that consecutive up-states are not correlated. From our simulations it emerges that the crucial features of this temporal evolution are (1) the different single neuron behavior in the two phases, namely the ability to oscillate between a very depolarized and hyperpolarized state, (2) the homeostatic mechanism driving activity in the up-state and (3) the network disfacilitation following up-states. The good agreement with experimental data indicates that the transition from an up-state to a down-state has a high degree of synchronization, whereas the onset of up-states is usually more gradual. According to our numerical results, the alternation between up and down-states is the expression of an homeostatic regulation which, during a burst, is activated to control the excitability of the system and avoid pathological behavior.

Finally we observe that the sequences of up and down-states generated by our model exhibit the same universal scaling functions found experimentally in rat cortex slices in vitro, namely a power law for the up-state and, for the down-state, a function peaked around a characteristic value with an exponentially decaying tail [20]. This point is particularly important because suggests that the lack of universality in the waiting time distribution for spike avalanches in anesthetized rats [33] could be due to the coexistence of different dynamic mechanisms, each one controlling ongoing activity at different temporal scales. Indeed, in freely behaving rats, where no down-states are observed, the waiting time distribution is controlled by the average occurrence rate [33], which, in terms of our model, is equivalent to $r_{u p}$, the average occurrence rate in the up-state. 


\section{References}

1. H. Berger, Arch. Psychiatr. Nervenkr. 87, 527 (1929)

2. G. Buzsaki, A. Draguhn, Science 304, 1926 (2004)

3. J.M. Beggs, D. Plenz, J. Neurosci. 23, 11167 (2003)

4. T. Petermann, T.C. Thiagarajan, M. Lebedev, M. Nicolelis, D.R. Chialvo, D. Plenz, PNAS 106(37), 15921 (2009)

5. O. Shriki, J. Alstott, F. Carver, T. Holroyd, R.N.A. Hanson, M.L. Smith, R. Coppola, E. Bullmore, D. Plenz, J. Neurosci. 33(16), 7079 (2013)

6. D. Fraiman, D.R. Chialvo, Front. Physiol. 3, 307 (2012)

7. E. Tagliazucchi, P. Balenzuela, D. Fraiman, D.R. Chialvo, Front. Physiol, 3, 15 (2012)

8. A. Haimovici, E. Tagliazucchi, P. Balenzuela, D.R. Chialvo, Phys. Rev. Lett. 110, 178101 (2013)

9. S. Scarpetta, A. de Candia, PLoS ONE 8(6), e64162 (2013)

10. T.H. Bullock, M.C. McClune, J.Z. Achimowicz, V.J. Iragui-Madoz, R.B. Duckrov, S.S. Spencer, Proc. Natl. Acad. Sci. USA 92, 11568 (1995)

11. T.H. Bullock, M.C. McClune, J.T. Enright, Neuroscience 121, 233 (2003)

12. E. Novikov, A. Novikov, D. Shannahoff-Khalsa, B. Schwartz, J. Wright, Phys. Rev. Lett. 97, $118102(2006)$

13. K.L. Hansen, V.V. Nikouline, J.M. Palva, R.J. IImoniemi, J. Neurosci. 21, 4 (2001)

14. C. Bedard, H. Kröger, A. Destexhe, Phys. Rev. Lett. 97, 118102 (2006)

15. N. Dehghani, C. Bedard, Sidney S. Cash, E. Halgren, A. Destexhe, J. Comput. Neurosci. $\mathbf{2 1}(29), 2010$

16. A. Corral, Phys. Rev. Lett. 92(10), 108501 (2004)

17. L. de Arcangelis, C. Godano, E. Lippiello, M. Nicodemi, Phys. Rev. Lett. 96, 051102 (2006)

18. R. Segev, B. Morris, E. Hulata, N. Cohen, A. Palevski, E. Kapon, Y. Shapira, E.B. Jacob, Phys. Rev. Lett. 88, 11 (2002)

19. F. Lombardi, H.J. Herrmann, C. Perrone-Capano, D. Plenz, L. de Arcangelis, Phys. Rev. Lett. 108, 228703 (2012)

20. F. Lombardi, H.J. Herrmann, D. Plenz, L. de Arcangelis, Frontiers in System Neuroscience (preprint) (2014)

21. L. de Arcangelis, C. Perrone-Capano, H.J. Herrmann, Phys. Rev. Lett. 96, 028107 (2006)

22. GL Pellegrini, L. de Arcangelis, H.J. Herrmann, C. Perrone-Capano, Phys. Rev. E 76, 028107 (2007)

23. R. Cossart, A. Aronov, R. Yuste, Nature 423, 283 (2003)

24. T.T.G. Hahn, B. Sakmann, M.R. Mehta, Nat. Neurosci. 9, 1359 (2006)

25. M.O. Cunningham, D.D. Pervouchine, C. Racca, N.J. Kopell, C.H. Davies, R.S.G. Jones, R.D. Traub, M.A. Whittington, PNAS 103, 5597 (2006)

26. B.J. He, J.M. Zempel, A.Z. Snyder, M.E. Raichle, Neuron 66, 353 (2010)

27. A. Mazzoni, F.D. Broccard, E. Garcia-Perez, P. Bonifazi, E.M. Ruaro, V. Torre, PLoS ONE 2(5), e439 (2007)

28. V. Pasquale, P. Massobrio, L.L. Bologna, M. Chiappalone, S. Martinoia, J. Neurosci. 153, $1354(2008)$

29. D.E. Gireesh, D. Plenz, PNAS 105(21), 7576 (2008)

30. D. Millman, S. Mihalas, A. Kirkwood, E. Niebur, Nat. Phys. 6, 801 (2010)

31. A. Bragin, G. Jandoó, Z. Nadasdy, J. Hetke, K. Wise, G. Buzsaki, J. Neurosci. 15, 47 (1995)

32. A. Corral, Phys. Rev. Lett. 95(2), 028501 (2005)

33. T.L. Ribeiro, M. Copelli, F. Caixeta, H. Belchior, D.R. Chialvo, PLoS ONE 5, e14129 (2010)

34. V.M. Eguiluz, D. Chialvo, G.A. Cecchi, M. Baliki, A.V. Apkarian, Phys. Rev. Lett. 94, $018102(2005)$

35. L. de Arcangelis, H.J. Herrmann, Front. Physio. 3, 62 (2012)

36. K.J. Staley, M. Longacher, J.S. Bains, A. Yee, Nat. Neurosci. 1, 201 (1998)

37. S.M. Thompson, H.L. Haas, B.H. Ghwiler, J. Physiol. 451, 347 (1992) 
38. M.V. Sanchez-Vives, L.G. Novak, D.A. McCormick, J. Neurosci. 20, 4286 (2000)

39. E. Maeda, H.P. Robinson, A. Kawana, J. Neurosci. 15, 6834 (1995)

40. C. Wilson, Scholarpedia J. 3(6), 1410 (2008)

41. I. Timofeev, F. Grenier, M. Steriade, PNAS 98(4), 1924 (2001)

42. W. Shew, H. Yang, T. Petermann, R. Roy, D. Plenz, J. Neurosci. 29(49), 15595 (2009)

43. D. Plenz, A. Aertsen, Neuroscience 70, 893 (1996)

44. W.H. Press, Astrophys. 7, 103 (1978)

45. R.F. Voss, J. Clark, Nature 258, 317 (1975)

46. W.S. Pritchard, Int. J. Neurosci. 66, 119 (1992)

47. C. Tang, P. Bak, K. Wiesenfeld, Phys. Rev. A 38, 1 (1988)

48. L. Laurson, M.J. Alava, S. Zapperi, J. Stat. Mech. L11001 (2005)

49. E. Lippiello, de Arcangelis, C. Godano, Phys. Rev. Lett. 100, 038501 (2008)

50. G. Boffetta, V. Carbone, P. Giuliani, P. Veltri, A. Vulpiani, Phys. Rev. Lett. 83(22), $4662(1999)$

51. T. Utsu, International Handbook of Earthquake and Engineering Seismology, Vol. 81A (Academic Press, 2002)

52. P. De Los Rios, Y.-C. Zhang, Phys. Rev. Lett. 82(3), 472 (1999)

53. R. Sanchez, D.E. Newman, D.A. Carreras, Phys. Rev. Lett. 88(6), 068302 (2002) 